\title{
Commentary on "Weiner et al., 2017, The tolerance and safety of 4-Demethyl-4-Cholesteryloxypenclomedine [DM-CHOC-PEN] in Adolescent and Young Adult (AYA) subjects with advanced malignancies"
}

\author{
Lee Roy Morgan* \\ DEKK-TEC, Inc., New Orleans, LA, USA
}

Malignancies of the central and spinal nervous systems (CNS \& SNS) occur at every age level of human life; no age group is spared the possibility of developing a primary or secondary CNS/SNS malignancy [1].

Almost 700,000 people in the US are living with tumors in the CNS or SNS tumors [1-3]. An additional 400,000 individuals are estimated to be living in other parts of the world with malignancies in the CNS or SNS [2]. Although the age range that is most affected by CNS and SNS malignancies are the 50-70s, no age range is spared [1].

The National Cancer Institute and the American Cancer Society in conjunction with the World Health Organization (WHO), EORTC, ECCO and UK Cancer Foundation estimate that nearly 15\% of CNS and SNS tumors worldwide involve the adolescent/young adult (AYA) age group, 15-39 years of age [2]. It is predicted that in the US alone 10,617 AYA aged individuals will be diagnosed with brain or CNS tumors resulting in 434 deaths this year [1].

Pediatric aged individuals with histories of cancer, who appeared to have 'beaten the odds' from previous malignancies are now, as AYAs, developing reoccurrences as well as new primary cancers involving the CNS and SNS after years of remission in increasing frequencies that have sharply increased since 1989 [2,3]. The most common types of cancer involving the CNS and SNS that the AYA population experience are primary brain tumors (glioblastoma (GBM), astrocytoma, etc.) and metastatic cancers - melanoma, leukemia and sarcoma [4].

For both male and female individuals $<20$ years of age, primary and secondary cancers of the CNS and SNS are the most common causes of death from cancer; and in the 20-39 year age group, they are the first cause of cancer-related deaths in males and the fifth cause of cancer-related deaths in females [2]. Over all in the 15- to 39-year old range - 5-year survivals have remained stagnant since 1075, and for individuals aged 30 to 34 , survival rates have actually decreased [2]. The incidence and histology of cancer types does vary according to subject age and gender $[2,3]$.

Results from surgery/radiation for localized non-invasive cancer are encouraging for all ages, including AYAs. However, for advanced disease unless a tumor possesses a phenotypic target or a genetic mutation, the long term outlook for survival beyond 1 year and quality of life beyond are limited [5]. Yes, standard of care - chemotherapy and radiation provide responses with improved survival, however, the long range prognosis is still not $100 \%$ [6]. Unfortunately, all ages of individuals with advanced CNS involvement do not have a very good prognosis [6].

The AYA age group of individuals with malignancies deserves special attention since they generally lack histories of comorbidities - hypertension, pulmonary, hepatic, etc. and may tolerate drugs differently from older subjects, but may still be at risk for toxicity with immuno/chemotherapy regimens in current use. AYA individuals with cancer also demonstrate different host biology, tumor pathophysiology and metabolize chemotherapy drugs differently than do either younger or older individuals $[2,6]$.

Weiner et al. presented early Phase I results and experiences with 4-demethyl-4-cholesteryl-oxypenclomedine (DM-CHOC-PEN) as treatment for cancers involving the CNS in AYA individuals [5]. Encouraging responses without Gr-3/4 toxicities for DM-CHOC-PEN in AYA subjects are reviewed in table 1.

Table 1 reviews the AYA subjects involved in the Phase I trial, their i. v. doses $\left(39,55\right.$, or $97.8 \mathrm{mg} / \mathrm{m}^{2} \mathrm{DM}$-CHOC-PEN) administered once every 21 days), their responses, and toxicities. To date, twelve subjects have been treated in the AYA age group; eleven subjects had cancer involving the CNS. Several subjects were treated at more than 1-dose level. The AYA subjects had advanced, chemo-resistant stage IV cancer - melanoma, NSCLC, breast, acute lymphocytic leukemia, oligodendroglioma and astrocytoma [7].

Unlike other penclomedines (e.g., PEN, NSC 338720, figure 1), DM-CHOC-PEN is non-neurotoxic [8]. It does cross the blood brain barrier $(\mathrm{BBB})$ in animals and humans with responses observed in sarcoma, astrocytoma, melanoma, ALL, lung, and breast cancers involving the spinal and central nervous systems in the settings of our clinical trial [8]. The drug accumulated (and was quantitated - 61$120 \mathrm{ng} / \mathrm{g}$ of tumor tissue) in human sarcoma and lung cancer tissues involving the CNS/SNS, but was not found in adjacent normal brain/ spinal tissue from subjects in Phase I/II trials [8].

${ }^{\star}$ Correspondence to: Lee Roy Morgan, DEKK-TEC, Inc., New Orleans, LA, USA, Tel: 504-583-6135; E-mail: 1rm1579@aol.com

Received: September 14, 2018; Accepted: September 24, 2018; Published: September 28, 2018 
Morgan LR (2018) Commentary on "Weiner et al., 2017, The tolerance and safety of 4-Demethyl-4-Cholesteryloxypenclomedine [DM-CHOC-PEN] in Adolescent and Young Adult (AYA) subjects with advanced malignancies"

Table 1. AYA subjects with advanced cancers treated with IV DM-CHOC-PEN ${ }^{+}$

\begin{tabular}{|c|c|c|c|c|c|}
\hline Cancer Type (\#) & Age/Sex & Dose $\left(\mathrm{mg} / \mathrm{m}^{2}\right)$ & Responders w/ CNS (\#) & OS (w/CNS) (mos) & Toxicity \\
\hline Melanoma (1) & $39 / \mathrm{F}$ & 98.7 & 0 & 2 & None \\
\hline NSCLC (1) & $39 / \mathrm{F}$ & 98.7 & 1 & $30+$ & None \\
\hline Breast (2) & $33 \& 29 / F$ & $39,55 \& 98.7$ & 1 & $12 \& 3$ & None \\
\hline Astrocytoma (1) & $37 / \mathrm{M}$ & 98.7 & 1 & $30+$ & None \\
\hline $\begin{array}{l}\text { ALL (1) } \\
\text { NHL (1) }\end{array}$ & $\begin{array}{l}\mathrm{M} / 39 \\
\mathrm{~F} / 28^{*}\end{array}$ & $\begin{array}{l}98.7 \\
98.7\end{array}$ & $\begin{array}{c}1 \\
\mathrm{NR}\end{array}$ & $\begin{array}{l}8 * * \\
\text { None }\end{array}$ & $\begin{array}{l}\text { None } \\
\text { None }\end{array}$ \\
\hline GBM (2) & $\begin{array}{l}F / 34 \\
F / 34\end{array}$ & $\begin{array}{l}85.8 \\
98.7\end{array}$ & $\begin{array}{l}\text { NR } \\
\text { NR }\end{array}$ & $\begin{array}{l}4 \\
2\end{array}$ & $\begin{array}{l}\text { None } \\
\text { None }\end{array}$ \\
\hline Oligoastrocytoma (1) & $\mathrm{M} / 39$ & 98.7 & NR & 3 & None \\
\hline
\end{tabular}<smiles>[R]Oc1c(Cl)c(OC)nc(C(Cl)(Cl)Cl)c1Cl</smiles>

Figure 1. Penclomedine analogs $-\mathrm{PEN}\left(\mathrm{R}=\mathrm{CH}_{3}\right)$; $\mathrm{DM}-\mathrm{PEN}(\mathrm{R}=\mathrm{H})$; DM-CHOC-PEN $(\mathrm{R}=$ $\mathrm{CO}_{2}$-cholesteryl)

DM-CHOC-PEN does not require hepatic activation and is active in nanogram quantities in vitro against melanoma GBM, non-small cell lung cancer (NSCLC) and breast cancer explants [8,9]. The drug does not require hepatic activation, which is in contrast to DM-PEN (Figure 1) and other analogs [8]. These observations have led to a proposed mechanism whereby DM-CHOC-PEN associates with erythrocyte membrane surfaces, penetrates the BBB and brain parenchyma and is transported into intracerebral tumors with L-glutamine, with which it shares common structural moieties [8]. Thus, DM-CHOC-PEN may be killing micro-metastases, inhibiting DNA repair (an alkylating agent), and inducing an 'abscopal' immune-type effect $[8,9]$.

The pharmacokinetic profile for DM-CHOC-PEN in the AYAs vs. 50-60 y/o adults supports the influence of individual comorbidity on drug metabolism; for the former $v s$. latter $-\mathrm{C}_{\text {max }}-60: 41 \mu \mathrm{g} / \mathrm{mL}$; AUC - 13,008:10,687 $\mu \mathrm{g} . \mathrm{h} / \mathrm{mL}$ and $\mathrm{T}_{1 / 2} \beta-28: 71 \mathrm{~h}$. The higher $\mathrm{C}_{\max }$, higher AUC (total drug available) and lower $\mathrm{T}_{1 / 2} \beta$ (rate of degradation) values for the AYA subjects reflects a 'healthier' metabolic profile for the drug than exists in the older adults that may have been receiving other medications for associated comorbidities resulting in induced hepatic metabolic activity.

Moreover, AYA subjects - 15-39 years old - are of major interest since they are not commonly enrolled in clinical trials and typically managed by pediatric and/or adult oncologist, rather than AYA oncology specialists that also appreciate the issues they face - physical, psychosocial, emotional, sexual, spiritual, financial, dietary, etc. and able to translate knowledge to them $[3,8]$.

A Phase II clinical trial with DM-CHOC-PEN in AYA subjects (15-39 years old) with malignancies involving the CNS is in progress to validate and expand the observations in table 1 [IND 68,876].

\section{Acknowledgements}

This research was supported by the following grants - NCI/SBIR grants - R43/44CA132257; R43CA203351; LACATS - U54M104940-1.

\section{References}

1. Siegel RL, Miller KD, Jemal A (2017) Cancer Statistics, 2017. CA Cancer J Clin 67: 7-30. [Crossref]

2. Hayes-Lattin H (2016) Integrating AYA oncology care into the worlds of pediatric and adult oncology care to improve cancer outcomes. The ASCO Post 10: 82-83.

3. Wilson E (2016) Brain tumors affect adolescents and young adults differently. Hem Onc today 8-9: 2016

4. Toronezos ES (2017) Discussions on adolescent and young adult survivorship. 2017 Cancer Survivor-ship Symposium.

5. Weiner RS, Ware ML, Bhandari T, Friedlander P, Morgan LR (2017) The Tolerance and Safety of 4-Demethyl-4-cholesteryloxypenclomedine [DM-CHOC-PEN] in Adolescent and Young Adult (AYA) Subjects with Advanced Malignancies. J Transl Sci 3: 1-4.

6. Franklin ARK (2016) A Growing identity for adolescent and young adult oncology Oncology Times 38: 8-10.

7. Doyle C (2017) What do we still need to know about adolescent and young adult survivorship? Oncol Pract Manag 17: 34-35.

8. Morgan LR, Struck RF, Waud WR, Jursic BS, Serota D, et al. (2009) Carbonate and carbamate derivatives of 4-demethylpenclomedine as novel anticancer Agents. Canc Chemo Pharmacol 64: 618-623. [Crossref]

9. Weiner RS, Friedlander P, Gordon C, Saenger Y, Ware RL, et al. (2016) Results of Phase II cancer clinical trials for 4-demethyl-4-cholesteryoxycarbonylpenclomedine (DM-CHOC-PEN). Proc Am Assoc Cancer Res 58: 236.

Copyright: (C2018 Morgan LR. This is an open-access article distributed under the terms of the Creative Commons Attribution License, which permits unrestricted use, distribution, and reproduction in any medium, provided the original author and source are credited. 